\title{
Fixed point theorems for mappings satisfying contractive conditions of integral type and applications
}

\author{
Zeqing Liu', Xin $\mathrm{Li}^{1}$, Shin Min Kang ${ }^{2^{*}}$ and Sun Young Cho ${ }^{2^{*}}$
}

\author{
* Correspondence: smkang@gnu. \\ ac.kr; ooly61@yahoo.co.kr \\ ${ }^{2}$ Department of Mathematics and \\ RINS, Gyeongsang National \\ University, Jinju 660-701, Korea \\ Full list of author information is \\ available at the end of the article
}

\begin{abstract}
In this paper, the existence, uniqueness and iterative approximations of fixed points for contractive mappings of integral type in complete metric spaces are established. As applications, the existence, uniqueness and iterative approximations of solutions for a class of functional equations arising in dynamic programming are discussed. The results presented in this paper extend and improve essentially the results of Branciari (A fixed point theorem for mappings satisfying a general contractive condition of integral type. Int. J. Math. Math. Sci. 29, 531-536, 2002), Kannan (Some results on fixed points. Bull. Calcutta Math. Soc. 60, 71-76, 1968) and several known results. Four concrete examples involving the contractive mappings of integral type with uncountably many points are constructed.
\end{abstract}

2010 Mathematics Subject Classfication: 54H25, 47H10, 49L20, 49L99, 90C39

Keywords: contractive mappings of integral type, complete metric space, fixed point theorem, functional equation, dynamic programming, bounded solution

\section{Introduction}

Throughout this paper, we assume that $\mathbb{R}=(-\infty,+\infty), \mathbb{R}^{+}=[0,+\infty), \mathbb{N}$ denotes the set of all positive integers, opt stands for sup or inf, $Z$ and $Y$ are Banach spaces, $S \subseteq Z$ is the state space, $D \subseteq Y$ is the decision space, $B(S)$ denotes the Banach space of all bounded real-valued functions on $S$ with norm

$$
\|g\|=\sup \{|g(x)|: x \in S\} \text { for any } g \in B(S)
$$

and

$$
\begin{gathered}
\Phi=\left\{\varphi: \varphi: \mathbb{R}^{+} \rightarrow \mathbb{R}^{+} \text {satisfies that } \varphi\right. \text { is Lebesgue integrable, } \\
\text { summable on each compact subset of } \mathbb{R}^{+} \\
\text {and } \left.\int_{0}^{\varepsilon} \varphi(t) d t>0 \text { for each } \varepsilon>0\right\}
\end{gathered}
$$

The famous Banach contraction principle is as follows.

Theorem 1.1. ([1]) Let $f$ be a mapping from a complete metric space $(X, d)$ into itself satisfying

$$
d(f x, f y) \leq c d(x, y), \quad \forall x, y \in X,
$$

\section{Springer}

(C) 2011 Liu et al; licensee Springer. This is an Open Access article distributed under the terms of the Creative Commons Attribution License (http://creativecommons.org/licenses/by/2.0), which permits unrestricted use, distribution, and reproduction in any medium, provided the original work is properly cited. 
where $c \in(0,1)$ is a constant. Then $f$ has a unique fixed point $a \in X$ such that $\lim _{n \rightarrow \infty} f^{n} x=$ a for each $x \in X$.

It is well known that the Banach contraction principle has a lot of generalizations and various applications in many directions, see, for example, [2-30] and the references cited therein. In 1962, Rakotch [29] extended the Banach contraction principle with replacing the contraction constant $c$ in (1.1) by a contraction function $\gamma$ and established the result later.

Theorem 1.2. ([29]) Let $f$ be a mapping from a complete metric space $(X, d)$ into itself satisfying

$$
d(f x, f y) \leq \gamma(d(x, y)) d(x, y), \quad \forall x, y \in X,
$$

where $\gamma: \mathbb{R}^{+} \rightarrow[0,1)$ is monotonically decreasing. Then $f$ has a unique fixed point $a \in$ $X$ such that $\lim _{n \rightarrow \infty} f^{n} x=a$ for each $x \in X$.

In 1968, Kannan [12] generalized the Banach contraction principle from continuous mappings to noncontinuous mappings and proved the following fixed point theorem.

Theorem 1.3. ([12]) Let $f$ be a mapping from a complete metric space $(X, d)$ into itself satisfying

$$
d(f x, f y) \leq c[d(x, f x)+d(y, f y)], \quad \forall x, y \in X,
$$

where $c \in\left(0, \frac{1}{2}\right)$ is a constant. Then $f$ has a unique fixed point in $X$.

In 2002, Branciari [8] gave an integral version of the Banach contraction principles and showed the following fixed point theorem.

Theorem 1.4. ([8]) Let $f$ be a mapping from a complete metric space $(X, d)$ into itself satisfying

$$
\int_{0}^{d(f x, f y)} \varphi(t) \mathrm{d} t \leq c \int_{0}^{d(x, y)} \varphi(t) \mathrm{d} t, \quad \forall x, y \in X,
$$

where $c \in(0,1)$ is a constant and $\phi \in \Phi$. Then $f$ has a unique fixed point $a \in X$ such that $\lim _{n \rightarrow \infty} f^{n} x=$ a for each $x \in X$.

In recent years, there has been increasing interest in the study of fixed points and common fixed points of mappings satisfying contractive conditions of integral type. The authors [2,3,9-11,28,30] and others continued the study of Branciari. In 2006, Aliouche [2] proved a fixed point theorem using a general contractive condition of integral type in symmetric spaces. In 2007, Djoudi and Aliouche [9] obtained common fixed point theorems of Gregus type for two pairs of weakly compatible mappings satisfying contractive conditions of integral type, and Suzuki [30] proved that Theorem 1.4 previously is a corollary of the Meir-Keeler fixed point theorem and that the Meir-Keeler contractions of integral type are still Meir-Keeler contractions. In 2009, Pathak [28] bore out a general common fixed point theorem of integral $\phi$-type for two pairs of weakly compatible mappings satisfying certain integral type implicit relations in symmetric spaces, and Jachymski [10] testified that most contractive conditions of integral type given recently by many authors coincide with classical ones and got a new contractive condition of integral type which is independent of classical ones. However, to the best of our knowledge, the concrete examples constructed in $[8,10]$, which guarantee the existence of fixed points for the contractive 
mappings of integral type in complete metric spaces, include at most countably many points.

On the other hand, by using various fixed point theorems, the authors [4-7,13-26] studied the existence, uniqueness and iterative approximations of solutions, coincidence solutions and nonnegative solutions for the functional equations arising in dynamic programming below

$$
\begin{aligned}
& f(x)=\inf _{y \in D}\{H(x, y, f(T(x, y)))\}, \quad \forall x \in Z, \\
& f(x)=\sup _{y \in D}\{H(x, y, f(T(x, y)))\}, \quad \forall x \in Z, \\
& f(x)=\operatorname{opt}_{y \in D}\{u(x, y)+H(x, y, f(T(x, y)))\}, \quad \forall x \in Z,
\end{aligned}
$$

where $x$ and $y$ signify the state and decision vectors, respectively, $T$ represents the transformation of the process, and $f(x)$ denotes the optimal return function with the initial state $x$.

The purposes of this paper are both to study the existence, uniqueness and iterative approximations of fixed points for three classes of contractive mappings of integral type, respectively, under different from or weaker than the conditions in $[1-3,8-11,28,30]$, to construct four examples with uncountably many points to show the superiority of the results presented in this paper and to show solvability of the functional Equation (1.7) in $B(S)$. Our results improve essentially Theorems 1.1-1.4.

\section{Lemmas}

The following lemmas play important roles in this paper.

Lemma 2.1. Let $\phi \in \Phi$ and $\left\{r_{n}\right\}_{n \in \mathbb{N}}$ be a nonnegative sequence with $\lim _{n \rightarrow \infty} r_{n}=a$. Then

$$
\lim _{n \rightarrow \infty} \int_{0}^{r_{n}} \varphi(t) \mathrm{d} t=\int_{0}^{a} \varphi(t) \mathrm{d} t .
$$

The proof of Lemma 2.1 follows from Remark 2.1 in [27].

Lemma 2.2. Let $\phi \in \Phi$ and $\left\{\mathrm{r}_{n}\right\}_{n \in \mathbb{N}}$ be a nonnegative sequence. Then $\lim _{n \rightarrow \infty} \int_{0}^{r_{n}} \varphi(t) \mathrm{d} t=0$ if and only if $\lim _{n \rightarrow \infty} r_{n}=0$.

The proof of Lemma 2.2 follows by Lemma 2.1 in [27].

Lemma 2.3. ([18]) Let $E$ be a set, $p$ and $q: E \rightarrow \mathbb{R}$ be mappings. If $\operatorname{opt}_{y \in E} p(y)$ and opt $_{y \in E} q(y)$ are bounded, then

$$
\left|\operatorname{opt}_{\gamma \in E} p(t)-\underset{\gamma \in E}{\operatorname{opt}} q(\gamma)\right| \leq \sup _{\gamma \in E}|p(\gamma)-q(\gamma)| .
$$

\section{Fixed point theorems for contractive mappings of integral type}

In this section, we show the existence, uniqueness and iterative approximations of fixed points for three classes of contractive mappings of integral type. For each $x \in X$ and $n \geq 0$, put $d_{n}=d\left(f^{n} x, f^{n+1} x\right)$.

Theorem 3.1. Let $f$ be a mapping from a complete metric space $(X, d)$ into itself satisfying 


$$
\int_{0}^{d(f x, f y)} \varphi(t) \mathrm{d} t \leq \alpha(d(x, y)) \int_{0}^{d(x, y)} \varphi(t) \mathrm{d} t, \quad \forall x, y \in X
$$

where $\phi \in \Phi$ and $\alpha: \mathbb{R}^{+} \rightarrow[0,1)$ is a function with

$$
\limsup _{s \rightarrow t} \alpha(s)<1, \quad \forall t>0 .
$$

Then $f$ has a unique fixed point $a \in X$ such that for each $x \in X, \lim _{n \rightarrow \infty} f^{n} x=a$. Proof. Let $x$ be an arbitrary point in $X$. It follows from (3.1) and (3.2) that

$$
\begin{aligned}
\int_{0}^{d_{n}} \varphi(t) \mathrm{d} t & =\int_{0}^{d\left(f^{n} x, f^{n+1} x\right)} \varphi(t) \mathrm{d} t \\
& \leq \alpha\left(d\left(f^{n-1} x, f^{n} x\right)\right) \int_{0}^{d\left(f^{n-1} x, f^{n} x\right)} \varphi(t) \mathrm{d} t \\
& =\alpha\left(d_{n-1}\right) \int_{0}^{d_{n-1}} \varphi(t) \mathrm{d} t \\
& \leq \int_{0}^{d_{n-1}} \varphi(t) \mathrm{d} t, \quad \forall n \in \mathbb{N} .
\end{aligned}
$$

Now, we show that

$$
d_{n} \leq d_{n-1}, \quad \forall n \in \mathbb{N} .
$$

Suppose that (3.4) does not hold. That is, there exists some $n_{0} \in \mathbb{N}$ satisfying

$$
d_{n_{0}}>d_{n_{0}-1} .
$$

Since $\phi \in \Phi$, it follows from (3.2), (3.3) and (3.5) that

$$
\begin{aligned}
0 & <\int_{0}^{d_{n_{0}}} \varphi(t) \mathrm{d} t \leq \int_{0}^{d_{n_{0-1}}} \varphi(t) \mathrm{d} t \leq \int_{0}^{d_{n_{0}}} \varphi(t) \mathrm{d} t \\
& \leq \alpha\left(d_{n 0-1}\right) \int_{0}^{d_{n_{0-1}}} \varphi(t) \mathrm{d} t<\int_{0}^{d_{n_{0-1}}} \varphi(t) \mathrm{d} t
\end{aligned}
$$

which means that

$$
\int_{0}^{d_{n_{0-1}}} \varphi(t) \mathrm{d} t<\int_{0}^{d_{n_{0-1}}} \varphi(t) \mathrm{d} t
$$

which is a contradiction and hence (3.4) holds. Note that (3.4) yields that the sequence $\left\{\mathrm{d}_{n}\right\}_{n \in \mathbb{N}}$ is nonincreasing, which implies that there exists a constant $c$ with $\lim _{n \rightarrow \infty} d_{\mathrm{n}}=c \geq 0$.

Next, we show that $c=0$. Otherwise $c>0$. Taking upper limit in (3.3) and using (3.2), Lemma 2.1 and $\phi \in \Phi$, we conclude that

$$
\begin{aligned}
0 & <\int_{0}^{c} \varphi(t) \mathrm{d} t \\
& =\limsup _{n \rightarrow \infty} \int_{0}^{d_{n}} \varphi(t) \mathrm{d} t \leq \limsup _{n \rightarrow \infty}\left(\alpha\left(d_{n-1}\right) \int_{0}^{d_{n-1}} \varphi(t) \mathrm{d} t\right) \\
& \leq \limsup _{n \rightarrow \infty} \alpha\left(d_{n-1}\right) \limsup _{n \rightarrow \infty} \int_{0}^{d_{n-1}} \varphi(t) \mathrm{d} t \\
& \leq\left(\limsup _{s \rightarrow c} \alpha(s)\right) \int_{0}^{c} \varphi(t) \mathrm{d} t \int_{0}^{c} \varphi(t) \mathrm{d} t,
\end{aligned}
$$


which is absurd. Therefore, $c=0$, that is,

$$
\lim _{n \rightarrow \infty} d_{n}=0
$$

Now, we claim that $\left\{f^{n} x\right\}_{n \in \mathbb{N}}$ is a Cauchy sequence. Suppose that $\left\{f^{n} x\right\}_{n \in \mathbb{N}}$ is not a Cauchy sequence, which means that there is a constant $\varepsilon>0$ such that for each positive integer $k$, there are positive integers $m(k)$ and $n(k)$ with $m(k)>n(k)>k$ such that

$$
d\left(f^{m(k)} x, f^{n(k)} x\right)>\varepsilon
$$

For each positive integer $k$, let $m(k)$ denote the least integer exceeding $n(k)$ and satisfying the above inequality. It follows that

$$
d\left(f^{m(k)} x, f^{n(k)} x\right)>\varepsilon \quad \text { and } \quad d\left(f^{m(k)-1} x, f^{n(k)} x\right) \leq \varepsilon, \quad \forall k \in \mathbb{N} .
$$

Note that $\forall k \in \mathbb{N}$

$$
\begin{aligned}
& d\left(f^{m(k)} x, f^{n(k)} x\right) \leq d\left(f^{n(k)} x, f^{m(k)-1} x\right)+d_{m(k)-1} \\
& \left|d\left(f^{m(k)} x, f^{n(k)+1} x\right)-d\left(f^{m(k)} x, f^{n(k)} x\right)\right| \leq d_{n(k)} \\
& \left|d\left(f^{m(k)+1} x, f^{n(k)+1} x\right)-d\left(f^{m(k)} x, f^{n(k)+1} x\right)\right| \leq d_{m(k)} \\
& \left|d\left(f^{m(k)+1} x, f^{n(k)+1} x\right)-d\left(f^{m(k)+1} x, f^{n(k)+2} x\right)\right| \leq d_{n(k)+1}
\end{aligned}
$$

In light of (3.6)-(3.8), we conclude that

$$
\begin{aligned}
\varepsilon & =\lim _{k \rightarrow \infty} d\left(f^{n(k)} x, f^{m(k)} x\right)=\lim _{k \rightarrow \infty} d\left(f^{m(k)} x, f^{n(k)+1} x\right) \\
& =\lim _{k \rightarrow \infty} d\left(f^{m(k)+1} x, f^{n(k)+1} x\right)=\lim _{k \rightarrow \infty} d\left(f^{m(k)+1} x, f^{n(k)+2} x\right) .
\end{aligned}
$$

In view of (3.1), we deduce that

$$
\begin{aligned}
& \int_{0}^{d\left(f^{m(k)+1} x, f^{n(k)+2} x\right)} \varphi(t) \mathrm{d} t \\
& \leq \alpha\left(d\left(f^{m(k)} x, f^{n(k)+1} x\right)\right) \int_{0}^{d\left(f^{m(k)} x, f^{n(k)+1} x\right)} \varphi(t) \mathrm{d} t, \quad \forall k \in \mathbb{N}
\end{aligned}
$$

Taking upper limit in (3.10) and by virtue of (3.2), (3.9), Lemma 2.1 and $\phi \in \Phi$, we get that

$$
\begin{aligned}
0 & <\int_{0}^{\varepsilon} \varphi(t) \mathrm{d} t=\limsup _{k \rightarrow \infty} \int_{0}^{d\left(f^{m(k)+1} x, f^{n(k)+2} x\right)} \varphi(t) \mathrm{d} t \\
& \leq \limsup _{k \rightarrow \infty} \alpha\left(d\left(f^{m(k)} x, f^{n(k)+1} x\right)\right) \limsup _{k \rightarrow \infty} \int_{0}^{d\left(f^{m(k)} x, f^{n(k)+1} x\right)} \varphi(t) \mathrm{d} t \\
& \leq\left(\limsup _{s \rightarrow \varepsilon} \alpha(s)\right) \int_{0}^{\varepsilon} \varphi(t) \mathrm{d} t \\
& <\int_{0}^{\varepsilon} \varphi(t) \mathrm{d} t,
\end{aligned}
$$

which is a contradiction. Thus, $\left\{f^{n} x\right\}_{n \in \mathbb{N}}$ is a Cauchy sequence. Since $(X, d)$ is a complete metric space, there exists a point $a \in X$ such that $\lim _{n \rightarrow \infty} f^{n} x=a$. By (3.1), (3.2) and Lemma 2.2, we arrive at 


$$
\begin{aligned}
0 & \leq \int_{0}^{d\left(f^{n+1} x, f a\right)} \varphi(t) \mathrm{d} t \leq \alpha\left(d\left(f^{n} x, a\right)\right) \int_{0}^{d\left(f^{n} x, a\right)} \varphi(t) \mathrm{d} t \\
& \leq \int_{0}^{d\left(f^{n} x, a\right)} \varphi(t) \mathrm{d} t \rightarrow 0 \quad \text { as } n \rightarrow \infty,
\end{aligned}
$$

which yields that

$$
\lim _{n \rightarrow \infty} \int_{0}^{d\left(f^{n+1} x, f a\right)} \varphi(t) \mathrm{d} t=0,
$$

which together with Lemma 2.2 gives that $\lim _{n \rightarrow \infty} d\left(f^{n+1} x, f a\right)=0$. Consequently, we conclude immediately that

$$
d(a, f a) \leq d\left(a, f^{n+1} x\right)+d\left(f^{n+1} x, f a\right) \rightarrow 0 \quad \text { as } n \rightarrow \infty,
$$

which means that $a=f a$.

Finally, we prove that $f$ has a unique fixed point in $X$. Suppose that $f$ has another fixed point $b \in X \backslash\{a\}$. It follows from $\phi \in \Phi$, (3.2) and (3.3) that

$$
\begin{aligned}
0 & <\int_{0}^{d(a, b)} \varphi(t) \mathrm{d} t=\int_{0}^{d(f a, f b)} \varphi(t) \mathrm{d} t \\
& \leq \alpha(d(a, b)) \int_{0}^{d(a, b)} \varphi(t) \mathrm{d} t<\int_{0}^{d(a, b)} \varphi(t) \mathrm{d} t,
\end{aligned}
$$

which is a contradiction. This completes the proof.

Theorem 3.2. Let $f$ be a mapping from a complete metric space $(X, d)$ into itself satisfying

$$
\begin{aligned}
\int_{0}^{d(f x, f y)} \varphi(t) \mathrm{d} t & \leq \alpha(d(x, y)) \int_{0}^{d(x, f x)} \varphi(t) \mathrm{d} t \\
& +\beta(d(x, y)) \int_{0}^{d(y, f y)} \varphi(t) \mathrm{d} t, \quad \forall x, y \in X,
\end{aligned}
$$

where $\phi \in \Phi$ and $\alpha, \beta: \mathbb{R}^{+} \rightarrow[0,1)$ are two functions with

$$
\begin{aligned}
& a(t)+\beta(t)<1, \quad \forall t \in \mathbb{R}^{+}, \quad \limsup _{s \rightarrow 0^{+}} \beta(s)<1, \\
& \limsup _{s \rightarrow t^{+}} \frac{\alpha(s)}{1-\beta(s)}<1, \quad \forall t>0 .
\end{aligned}
$$

Then $f$ has a unique fixed point $a \in X$ such that for each $x \in X, \lim _{n \rightarrow \infty} f^{n} x=a$.

Proof. Let $x$ be an arbitrary point in $X$. By (3.11), we obtain that

$$
\begin{aligned}
\int_{0}^{d_{n}} \varphi(t) \mathrm{d} t= & \int_{0}^{d\left(f^{n} x, f^{n+1} x\right)} \varphi(t) \mathrm{d} t \\
\leq & \alpha\left(d\left(f^{n-1} x, f^{n} x\right)\right) \int_{0}^{d\left(f^{n-1} x, f^{n} x\right)} \varphi(t) \mathrm{d} t \\
& +\beta\left(d\left(f^{n-1} x, f^{n} x\right)\right) \int_{0}^{d\left(f^{n} x, f^{n+1} x\right)} \varphi(t) \mathrm{d} t \\
= & \alpha\left(d_{n-1}\right) \int_{0}^{d_{n-1}} \varphi(t) \mathrm{d} t+\beta\left(d_{n-1}\right) \int_{0}^{d_{n}} \varphi(t) \mathrm{d} t, \quad \forall n \in \mathbb{N},
\end{aligned}
$$


which together with (3.12) yields that

$$
\int_{0}^{d_{n}} \varphi(t) d t \leq \frac{\alpha\left(d_{n-1}\right)}{1-\beta\left(d_{n-1}\right)} \int_{0}^{d_{n-1}} \varphi(t) \mathrm{d} t \leq \int_{0}^{d_{n-1}} \varphi(t) \mathrm{d} t, \quad \forall n \in \mathbb{N} .
$$

As in the proof of Theorem 3.1, we conclude similarly that the sequence $\left\{\mathrm{d}_{n}\right\}_{n \in \mathbb{N}}$ is nonincreasing and converges to 0 .

Next, we show that $\left\{f^{n} x\right\}_{n \in \mathbb{N}}$ is a Cauchy sequence. Suppose that $\left\{f^{n} x\right\}_{n \in \mathbb{N}}$ is not a Cauchy sequence. It follows that there is a constant $\varepsilon>0$ such that for each positive integer $k$, there are positive integers $m(k)$ and $n(k)$ with $m(k)>n(k)>k$ with

$$
d\left(f^{m(k)} x, f^{n(k)} x\right)>\varepsilon
$$

For each positive integer $k$, let $m(k)$ denote the least integer exceeding $n(k)$ and satisfying the above inequality. It is easy to verify that (3.7)-(3.9) hold. By means of (3.9), (3.11), (3.12), Lemma 2.1 and $\phi \in \Phi$, we get that

$$
\begin{aligned}
0< & \int_{0}^{\varepsilon} \varphi(t) \mathrm{d} t=\limsup _{k \rightarrow \infty} \int_{0}^{d\left(f^{m(k)+1} x, f^{n(k)+2} x\right)} \varphi(t) \mathrm{d} t \\
\leq & \limsup _{k \rightarrow \infty}\left(\alpha\left(d\left(f^{m(k)} x, f^{n(k)+1} x\right)\right) \int_{0}^{d_{m(k)}} \varphi(t) \mathrm{d} t\right. \\
& \left.+\beta\left(d\left(f^{m(k)} x, f^{n(k)+1} x\right)\right) \int_{0}^{d_{n(k)+1}} \varphi(t) \mathrm{d} t\right) \\
\leq & \limsup _{k \rightarrow \infty} \int_{0}^{d_{m(k)}} \varphi(t) \mathrm{d} t+\limsup _{k \rightarrow \infty} \int_{0}^{d_{n(k)+1}} \varphi(t) \mathrm{d} t \\
= & 0,
\end{aligned}
$$

which is a contradiction. Hence, $\left\{f^{n} x\right\}_{n \in \mathbb{N}}$ is a Cauchy sequence. Since $(X, d)$ is a complete metric space, there exists a point $a \in X$ such that $\lim _{n \rightarrow \infty} f^{n} x=a$, which means that $\lim _{n \rightarrow \infty} d\left(f^{n+1} x, f a\right)=d(a, f a)$. If $d(a, f a) \neq 0$, by (3.11), (3.12) and Lemma 2.1 , we infer that

$$
\begin{aligned}
0< & \int_{0}^{d(a, f a)} \varphi(t) d t=\limsup _{n \rightarrow \infty} \int_{0}^{d\left(f^{n+1} x, f a\right)} \varphi(t) d t \\
\leq & \limsup _{n \rightarrow \infty}\left(\alpha\left(d\left(f^{n} x, a\right)\right) \int_{0}^{d\left(f^{n} x, f^{n+1} x\right)} \varphi(t) d t\right) \\
& +\limsup _{n \rightarrow \infty}\left(\beta\left(d\left(f^{n} x, a\right)\right) \int_{0}^{d(a, f a)} \varphi(t) d t\right) \\
= & \left(\limsup _{s \rightarrow 0^{+}} \beta(s)\right) \int_{0}^{d(a, f a)} \varphi(t) d t \\
< & \int_{0}^{d(a, f a)} \varphi(t) d t,
\end{aligned}
$$

which is impossible. Thus, $d(a, f a)=0$. That is, $a=f a$.

Finally, we prove that $f$ has a unique fixed point in $X$. Suppose that $f$ has another fixed point $b \in X \backslash\{a\}$. It follows from $\phi \in \Phi$ and (3.12) that 


$$
\begin{aligned}
0 & <\int_{0}^{d(a, b)} \varphi(t) \mathrm{d} t=\int_{0}^{d(f a, f b)} \varphi(t) \mathrm{d} t \\
& \leq \alpha(d(a, b)) \int_{0}^{d(a, f a)} \varphi(t) \mathrm{d} t+\beta(d(a, b)) \int_{0}^{d(b, f b)} \varphi(t) \mathrm{d} t \\
& =0
\end{aligned}
$$

which is a contradiction. This completes the proof.

As in the proof of Theorem 3.2, we get similarly the below result.

Theorem 3.3. Let $f$ be a mapping from a complete metric space $(X, d)$ into itself satisfying

$$
\begin{aligned}
& \int_{0}^{d(f x, f y)} \varphi(t) \mathrm{d} t \\
& \leq \gamma(d(x, y))\left(\int_{0}^{d(x, f x)} \varphi(t) \mathrm{d} t+\int_{0}^{d(y, f y)} \varphi(t) \mathrm{d} t\right), \quad \forall x, y \in X,
\end{aligned}
$$

where $\phi \in \Phi$ and $\gamma: \mathbb{R}^{+} \rightarrow\left[0, \frac{1}{2}\right)$ is a function with

$$
\limsup _{s \rightarrow t^{+}} \frac{\gamma(s)}{1-\gamma(s)}<1, \quad \forall t>0 .
$$

Then $f$ has a unique fixed point $a \in X$ such that for each $x \in X, \lim _{n \rightarrow \infty} f^{n} x=a$.

\section{Remarks and illustrative examples}

In this section, by constructing four nontrivial examples with uncountably many points, we discuss and compare the fixed point theorems obtained in Section 3 with the known results in Section 1.

Remark 4.1. If $\alpha(t)=c$ for all $t \in \mathbb{R}^{+}$, where $c \in(0,1)$ is a constant, then Theorem 3.1 changes into Theorem 1.4; furthermore, if $\phi(t)=1$ for all $t \in \mathbb{R}^{+}$, then Theorem 3.1 brings Theorem 1.1. The following example manifests that Theorem 3.1 extends substantially Theorems 1.1 and 1.4 .

Example 4.1. Let $X=\mathbb{R}^{+}$be endowed with the Euclidean metric $d=|\cdot|, f: X \rightarrow X$, $\alpha: \mathbb{R}^{+} \rightarrow[0,1)$ and $\phi \in \Phi$ be defined by

$$
f(x)=\frac{x}{1+x}, \quad \forall x \in \mathbb{R}^{+}, \quad \varphi(t)=2 t, \quad \forall t \in \mathbb{R}^{+}
$$

and

$$
\alpha(t)=\left\{\begin{array}{l}
\frac{1}{2}, \quad t=0, \\
\frac{1}{(1+t)^{2}}, \forall t \in(0,+\infty) .
\end{array}\right.
$$

It is obvious that (3.2) holds and

$$
\begin{aligned}
\int_{0}^{d(f x, f y)} \varphi(t) \mathrm{d} t & =\left(\frac{x}{1+x}-\frac{y}{1+y}\right)^{2} \\
& =\frac{(x-y)^{2}}{(1+x)^{2}(1+y)^{2}} \leq \frac{(x-y)^{2}}{1+|x-y|)^{2}} \\
& =\alpha(d(x, y)) \int_{0}^{d(x, y)} \varphi(t) \mathrm{d} t, \quad \forall x, y \in X .
\end{aligned}
$$


That is, the conditions of Theorem 3.1 are fulfilled. It follows from Theorem 3.1 that $f$ has a unique fixed point $0 \in X$. But, we can neither invoke Theorem 1.1 nor Theorem 1.4 to show the existence of a fixed point of $f$ in $X$ because (1.1) and (1.4) do not hold.

Suppose that (1.1) holds. It follows that there exists a constant $c \in(0,1)$ satisfying

$$
d(f x, f y)=\frac{|x-y|}{(1+x)(1+y)} \leq c|x-y|, \quad \forall x, y \in X,
$$

which gives that

$$
\frac{1}{(1+x)(1+y)} \leq c, \quad \forall x, y \in X \text { with } x \neq y,
$$

which yields that $c \geq 1$, which is absurd.

Suppose that (1.4) holds. It follows that there exists some constant $c \in(0,1)$ satisfying

$$
\begin{aligned}
\int_{0}^{d(f x, f y)} \varphi(t) d t & =\left(\frac{x}{x+1}-\frac{y}{y+1}\right)^{2} \leq c(x-y)^{2} \\
& =c \int_{0}^{d(x, y)} \varphi(t) d t, \quad \forall x, y \in X,
\end{aligned}
$$

which yields that

$$
\frac{1}{(x+1)^{2}(y+1)^{2}} \leq c, \quad \forall x, y \in X \text { with } x \neq y,
$$

which means that

$$
1=\lim _{\substack{(x, y) \rightarrow(0,0) \\ x \neq y}} \frac{1}{(x+1)^{2}(y+1)^{2}} \leq c<1,
$$

which is a contradiction.

Remark 4.2. In case $\phi(t)=1$ for all $t \in \mathbb{R}^{+}$, then Theorem 3.1 reduces to a result, which generalizes Theorem 1.2. The following example reveals that Theorem 3.1 is a proper generalization Theorem 1.2.

Example 4.2. Let $X=\mathbb{R}^{+}$be endowed with the Euclidean metric $d=|\cdot|, f: X \rightarrow X$, $\alpha: \mathbb{R}^{+} \rightarrow[0,1)$ and $\phi \in \Phi$ be defined by

$$
f(x)=\frac{1}{1+x}, \quad \forall x \in \mathbb{R}^{+}, \quad \varphi(t)=2 t, \quad \forall t \in \mathbb{R}^{+}
$$

and

$$
\alpha(t)=\left\{\begin{array}{l}
\frac{1}{3}, \quad t=0, \\
\frac{1}{1+t,}, \forall t \in(0,1], \\
\frac{t}{1+t}, \forall t \in(1,+\infty) .
\end{array}\right.
$$

It is easy to see that (3.2) holds. In order to verify (3.1), we have to consider three possible cases as follows: 
Case 1. $x, y \in X$ with $x=y$. It is clear that

$$
\int_{0}^{d(f x, f y)} \varphi(t) \mathrm{d} t=0=\alpha(d(x, y)) \int_{0}^{d(x, y)} \varphi(t) \mathrm{d} t
$$

Case 2. $x, y \in X$ with $0<|x-y| \leq 1$. Note that

$$
\begin{aligned}
\int_{0}^{d(f x, f y)} \varphi(t) d t & =\left(\frac{1}{1+x}-\frac{1}{1+y}\right)^{2}=\frac{(x-y)^{2}}{(1+x)^{2}(1+y)^{2}} \\
& \leq \frac{(x-y)^{2}}{(1+x)(1+y)} \leq \frac{(x-y)^{2}}{1+|x-y|} \\
& =\alpha(d(x, y)) \int_{0}^{d(x, y)} \varphi(t) d t
\end{aligned}
$$

Case 3. $x, y \in X$ with $|x-y|>1$. It follows that

$$
\begin{aligned}
\int_{0}^{d(f x, f y)} \varphi(t) d t & =\left(\frac{1}{1+x}-\frac{1}{1+y}\right)^{2}=\frac{(x-y)^{2}}{(1+x)^{2}(1+y)^{2}} \\
& \leq \frac{(x-y)^{2}}{(1+x)(1+y)} \leq \frac{(x-y)^{2}}{1+|x-y|} \\
& \leq \frac{|x-y|^{3}}{1+|x-y|}=\alpha(d(x, y)) \int_{0}^{d(x, y)} \varphi(t) d t .
\end{aligned}
$$

Hence, (3.1) holds. Consequently, the conditions of Theorem 3.1 are satisfied.

It follows from Theorem 3.1 that $\mathrm{f}$ has a unique fixed point $\frac{\sqrt{5}-1}{2} \in X$.

However, Theorem 1.2 is useless in guaranteeing the existence of a fixed point of $f$ in $X$. Otherwise, suppose that the conditions of Theorem 1.2 are fulfilled. Notice that $\gamma: \mathbb{R}$ $+\rightarrow[0,1)$ is monotonically decreasing. It follows that $\lim _{t \rightarrow \infty+} \gamma(\mathrm{t})$ exists and belongs to $[\gamma(1), \gamma(0)] \subset[0,1)$. Using (1.2), we infer that

$$
\begin{aligned}
\frac{|x-y|}{(1+x)(1+y)} & =d(f x, f y) \leq \gamma(d(x, y)) d(x, y) \\
& =|x-y| \gamma(|x-y|), \quad \forall x, y \in X,
\end{aligned}
$$

which implies that

$$
\frac{1}{(1+x)(1+y)} \leq \gamma(|x-y|), \quad \forall x, y \in X \text { with } x \neq y,
$$

which yields that

$$
\begin{aligned}
1 & =\lim _{\substack{(x, y) \rightarrow(0,0) \\
x \neq y}} \frac{1}{(1+x)(1+\gamma)} \leq \lim _{\substack{(x, y) \rightarrow(0,0) \\
x \neq y}} \gamma(|x-\gamma|) \\
& =\lim _{t \rightarrow 0^{+}} \gamma(t) \leq \gamma(0)<1,
\end{aligned}
$$

which is impossible.

Remark 4.3. In case $\phi(t)=1$ and $\gamma(t)=h$ for all $t \in \mathbb{R}^{+}$, then Theorem 3.3 comes into being Theorem 1.3. The below example demonstrates that Theorem 3.3 is indeed a proper extension of Theorem 1.3. 
Example 4.3. Let $X=[0,4]$ be endowed with the Euclidean metric $d=|\cdot|, f: X \rightarrow X, \gamma: \mathbb{R}^{+} \rightarrow\left[0, \frac{1}{2}\right)$ and $\phi \in \Phi$ be defined by

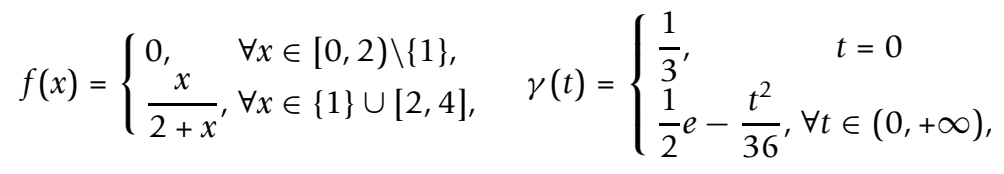

and

$$
\varphi(t)=e^{t}, \quad \forall t \in \mathbb{R}^{+},
$$

respectively. It is obvious that (3.14) holds and (3.13) is equivalent to

$$
e^{d(f x, f y)}-1 \leq \gamma(d(x, y))\left(e^{d(x, f x)}+e^{d(y, f y)}-2, \quad \forall x, y \in X .\right.
$$

Note that $x$ and $y$ in (4.1) are symmetric, (4.1) holds for all $x=y \in X$ and

$$
\gamma(d(x, y))<\frac{1}{2}, \quad \forall x, y \in X
$$

In order to verify (3.13), by (4.1) and (4.2) we need only to show that

$$
e^{d(f x, f y)} \leq \gamma(d(x, y))\left(e^{d(x, f x)}+e^{d(y, f y)}\right), \quad \forall x, y \in X \text { with } x>y .
$$

Now, we have to consider the below six possible cases:

Case 1. $x, y \in X$ with $4 \geq x>y \geq 2$. It follows that

$$
\begin{aligned}
& \frac{e^{d(f x, f y)}}{e^{d(x, f x)}+e^{d(y, f y)}}=\frac{e^{\frac{2(x-y)}{(2+x)(2+y)}}}{e^{\frac{x^{2}+x}{2+x}+\frac{y^{2}+y}{2+y}}} \leq \frac{e^{\frac{2(x-y)}{(2+x)(2+y)}}}{2 e^{\frac{x^{2}+x}{2(2+x)}} \frac{y^{2}+y}{2(2+y)}} \\
& =\frac{1}{2} e^{\frac{4(x-y)-\left[\left(x^{2}+x\right)(2+y)+\left(y^{2}+y\right)(2+x)\right]}{2(x+2)(y+2)}} \\
& =\frac{1}{2} e^{\frac{-2(x-y)^{2}+2 x-6 y(x+1)-x y(x+y)}{2(x+2)(y+2)}} \\
& \leq \frac{1}{2} e^{-\frac{(x-y)^{2}}{(x+2)(y+2)}} \leq \frac{1}{2} e^{-\frac{(x-y)^{2}}{36}}=\gamma(d(x, y)) ;
\end{aligned}
$$

Case 2. $x, y \in X$ with $4 \geq x \geq 2>\mathrm{y} \geq 0$ and $y \neq 1$. It is clear that

$$
\begin{aligned}
\frac{e^{d(f x, f y)}}{e^{d(x, f x)}+e^{d(y, f y)}} & =\frac{e^{\frac{x}{2+x}}}{e^{\frac{x^{2}+x}{2+x}}+e^{y}} \leq \frac{e^{\frac{x}{2+x}}}{2 e^{\frac{x^{2}+x}{2(2+x)}} e^{\frac{y}{2}}} \\
& =\frac{1}{2} e^{\frac{x-x^{2}-2 y-x y}{2(2+x)}} \leq \frac{1}{2} e^{\frac{x-x^{2}-2 y-x y}{12}} \\
& \leq \frac{1}{2} e^{-\frac{(x-y)^{2}}{36}}=\gamma(d(x, y))
\end{aligned}
$$


Case 3. $x ; y \in X$ with $4 \geq x \geq 2$ and $y=1$. It follows that

$$
\begin{aligned}
& \frac{e^{d(f x, f y)}}{e^{d(x, f x)}+e^{d(y, f y)}}=\frac{e^{\frac{2(x-1)}{3(2+x)}}}{e^{\frac{x^{2}+x}{2+x}}+e^{\frac{2}{3}}} \leq \frac{e^{\frac{2(x-1)}{3(2+x)}}}{2 e^{\frac{x^{2}+x}{2(2+x)}} e^{\frac{1}{3}}} \\
& =\frac{1}{2} e^{\frac{4(x-1)-\left[3\left(x^{2}+x\right)+2(2+x)\right]}{6(x+2}} \leq \frac{1}{2} e^{-\frac{(x-1)^{2}}{3(x+2)}} \\
& \leq \frac{1}{2} e^{-\frac{(x-y)^{2}}{36}}=\gamma(d(x, y)) ;
\end{aligned}
$$

Case 4. $x, y \in X$ with $2>x>y \geq 0, x \neq 1$ and $y \neq 1$. Notice that

$$
\begin{aligned}
\frac{e^{d(f x, f y)}}{e^{d(x, f x)}+e^{d(y, f y)}} & =\frac{e^{0}}{e^{x}+e^{y}} \leq \frac{1}{2 e^{\frac{x}{2}} e^{\frac{y}{2}}}=\frac{1}{2} e^{-\frac{x+y}{2}} \\
& \leq \frac{1}{2} e^{-\frac{(x-y)^{2}}{36}}=\gamma(d(x, y)) ;
\end{aligned}
$$

Case 5. $x, y \in X$ with $x=1>y \geq 0$. Obviously

$$
\begin{aligned}
\frac{e^{d(f x, f y)}}{e^{d(x, f x)}+e^{d(y, f y)}} & =\frac{e^{\frac{1}{3}}}{e^{\frac{2}{3}}+e^{y}} \leq \frac{e^{\frac{1}{3}}}{e^{\frac{2}{3}}+1}<\frac{(2.72)^{\frac{1}{3}}}{(2.718)^{\frac{2}{3}}+1} \\
& <\frac{1.396}{2.94}<\frac{1}{2 \times 1.05}<\frac{1}{2} e^{-\frac{1}{36}} \\
& \leq \frac{1}{2} e^{-\frac{(x-y)^{2}}{36}}=\gamma(d(x, y))
\end{aligned}
$$

Case 6. $x, y \in X$ with $2>x>1=y$. Notice that

$$
\begin{aligned}
\frac{e^{d(f x, f y)}}{e^{d(x, f x)}+e^{d(y, f y)}} & =\frac{e^{\frac{1}{3}}}{e^{x}+e^{\frac{2}{3}}}<\frac{e^{\frac{1}{3}}}{e+e^{\frac{2}{3}}} \leq \frac{e^{\frac{1}{3}}}{2 e^{\frac{1}{2}} e^{\frac{1}{3}}}=\frac{1}{2} e^{-\frac{1}{2}} \\
& <\frac{1}{2} e^{-\frac{1}{36}} \leq \frac{1}{2} e^{-\frac{(x-y)^{2}}{36}}=\gamma(d(x, y)) .
\end{aligned}
$$

Hence, (3.13) holds. That is, the conditions of Theorem 3.3 are satisfied. It follows from Theorem 3.3 that $f$ has a unique fixed point in $X$. However, it is easy to verify that for $x_{0}=1$ and $y_{0}=0$

$$
d\left(f x_{0}, f y_{0}\right)=\frac{1}{3} \nless \frac{2 c}{3}=c\left(d\left(x_{0}, f x_{0}\right)+d\left(y_{0}, f y_{0}\right)\right), \quad \forall c \in\left[0, \frac{1}{2}\right),
$$

which yields that (1.3) in Theorem 1.3 does not hold.

Next, we construct an example with uncountably many points to explain Theorem 3.2 . 
Example 4.4. Let $X=[1,3]$ be endowed with the Euclidean metric $d=|; \cdot|, f: X \rightarrow$ $X, \alpha, \beta: \mathbb{R}^{+} \rightarrow[0,1)$ and $\phi \in \Phi$ be defined by

$$
f(x)=\left\{\begin{array}{l}
1, \forall x \in[1,2) \\
\frac{x}{2}, \forall x \in[2,3]
\end{array}\right.
$$

and

$$
\varphi(t)=2 t, \quad \alpha(t)=\frac{t^{2}}{(1+t)^{2}}, \beta(t)=\frac{2 t}{(1+t)^{2 \prime}} \quad \forall t \in \mathbb{R}^{+} .
$$

It is easy to see that (3.12) holds. In order to verify (3.11), we have to consider the below five possible cases:

Case 1. $x, y \in X$ with $3 \geq x \geq y \geq 2$. Note that

$$
\begin{aligned}
\int_{0}^{d(f x, f y)} \varphi(t) d t= & \frac{(x-y)^{2}}{4} \leq \frac{(x-y)^{2}}{(1+x-y)^{2}} \frac{x^{2}}{4} \\
\leq & \alpha(d(x, y)) \int_{0}^{d(x, f x)} \varphi(t) d t \\
\leq & \alpha(d(x, y)) \int_{0}^{d(x, f x)} \varphi(t) d t \\
& +\beta(d(x, y)) \int_{0}^{d(y, f y)} \varphi(t) d t
\end{aligned}
$$

Case 2. $x, y \in X$ with $x \in[2,3]$ and $y \in[1,2)$. It follows that

$$
\begin{aligned}
\int_{0}^{d(f x, f y)} \varphi(t) d t= & \left(\frac{x}{2}-1\right)^{2} \leq \frac{(x-y)^{2}}{4} \leq \frac{(x-y)^{2}}{(1+x-y)^{2}} \frac{x^{2}}{4} \\
\leq & \alpha(d(x, y)) \int_{0}^{d(x, f x)} \varphi(t) d t \\
\leq & \alpha(d(x, y)) \int_{0}^{d(x, f x)} \varphi(t) d t \\
& +\beta(d(x, y)) \int_{0}^{d(y, f y)} \varphi(t) d t
\end{aligned}
$$

Case 3. $x, y \in X$ with $x, y \in[1,2)$. Notice that $f x=f y=1$. It follows that

$$
\begin{aligned}
\int_{0}^{d(f x, f y)} \varphi(t) \mathrm{d} t= & \leq \alpha(d(x, y)) \int_{0}^{d(x, f x)} \varphi(t) \mathrm{d} t \\
& +\beta(d(x, y)) \int_{0}^{d(y, f y)} \varphi(t) \mathrm{d} t, \quad \forall x, y \in X ;
\end{aligned}
$$

Case 4. $x, y \in X$ with $3 \geq y>x \geq 2$. Note that

$$
\begin{aligned}
\int_{0}^{d(f x, f y)} \varphi(t) d t= & \frac{(y-x)^{2}}{4} \leq \frac{y-x}{2} \leq \frac{2(y-x)}{(1+y-x)^{2}} \frac{y^{2}}{4} \\
= & \beta(d(x, y)) \int_{0}^{d(y, f y)} \varphi(t) d t \\
\leq & \alpha(d(x, y)) \int_{0}^{d(x, f x)} \varphi(t) d t \\
& +\beta(d(x, y)) \int_{0}^{d(y, f y)} \varphi(t) d t
\end{aligned}
$$


Case 5. $x, y \in X$ with $x \in[1,2)$ and $y \in[2,3]$. Note that

$$
\begin{aligned}
\int_{0}^{d(f x, f y)} \varphi(t) \mathrm{d} t= & \left(\frac{y}{2}-1\right)^{2} \leq \frac{y-x}{2} \leq \frac{2(y-x)}{(1+y-x)^{2}} \frac{y^{2}}{4} \\
\leq & \beta(d(x, y)) \int_{0}^{d(y, f y)} \varphi(t) \mathrm{d} t \\
\leq & \alpha(d(x, y)) \int_{0}^{d(x, f x)} \varphi(t) \mathrm{d} t \\
& +\beta(d(x, y)) \int_{0}^{d(y, f y)} \varphi(t) \mathrm{d} t
\end{aligned}
$$

that is, (3.11) holds. Thus, all the conditions of Theorem 3.2 are satisfied. It follows from Theorem 3.2 that $f$ has a unique fixed point $1 \in X$.

\section{Applications}

In this section, by using the fixed point theorems obtained in Section 3, we study solvability of the functional Equation $(1.7)$ in $B(S)$.

Theorem 5.1. Let $u: S \times D \rightarrow \mathbb{R}, T: S \times D \rightarrow S, H: S \times D \times \mathbb{R} \rightarrow \mathbb{R}, \phi \in \Phi$ and $\alpha$ : $\mathbb{R}^{+} \rightarrow[0,1)$ satisfy $(3.2)$,

$$
u \text { and } H \text { are bounded }
$$

and

$$
\begin{aligned}
& \int_{0}^{|H(x, y, g(T(x, y)))-H(x, y, h(T(x, y)))|} \varphi(t) \mathrm{d} t \leq \alpha(\|g-h\|) \int_{0}^{\|g-h\|} \varphi(t) \mathrm{d} t, \\
& \forall(x, y, g, h) \in S \times D \times B(S) \times B(S) .
\end{aligned}
$$

Then the functional Equation (1.7) has a unique solution $w \in B(S)$ and $\left\{A^{n} z\right\}_{n \in \mathbb{N}}$ converges to $w$ for each $z \in B(S)$, where the mapping $A$ is defined by

$$
A z(x)=\underset{y \in D}{\operatorname{opt}}\{u(x, y)+H(x, y, z(T(x, y)))\}, \quad \forall x \in S .
$$

Proof. It follows from (5.1) that there exists $M>0$ satisfying

$$
\sup \{|u(x, y)|,|H(x, y, t)|:(x, y, t) \in S \times D \times \mathbb{R}\} \leq M
$$

It is easy to see that $A$ is a self-mappings in $B(S)$ by (5.3), (5.4) and Lemma 2.3.

Using Theorem 12.34 in [31] and $\phi \in \Phi$, we conclude that for each $\varepsilon>0$, there exists $\delta>0$ satisfying

$$
\int_{C} \varphi(t) \mathrm{d} t<\varepsilon, \quad \forall C \subseteq[0,2 M] \text { with } m(C) \leq \delta,
$$

where $m(C)$ denotes the Lebesgue measure of $C$.

Let $x \in S, h, g \in B(S)$. Suppose that opt ${ }_{y \in D}=\inf _{y \in D}$. Clearly, (5.3) implies that there exist $y, z \in D$ satisfying

$$
\begin{aligned}
& A g(x)>u(x, y)+H(x, y, g(T(x, y)))-\delta \\
& A h(x)>u(x, z)+H(x, z, h(T(x, z)))-\delta \\
& A g(x) \leq u(x, z)+H(x, z, g(T(x, z))) \\
& A h(x) \leq u(x, y)+H(x, y, h(T(x, z)))
\end{aligned}
$$


Put

$$
\begin{array}{ll}
H_{1}=H(x, y, g(T(x, y))), & H_{2}=H(x, y, h(T(x, y))), \\
H_{3}=H(x, z, g(T(x, z))), & H_{4}=H(x, z, h(T(x, z))) .
\end{array}
$$

It is easy to verify that

$$
\begin{aligned}
A g(x)-A h(x)> & H(x, z, g(T(x, y)))-H(x, y, h(T(x, z)))-\delta \\
\geq & -\max \{|H(x, y, g(T(x, y)))-H(x, y, h(T(x, y)))|, \\
& |H(x, z, g(T(x, z)))-H(x, z, h(T(x, z)))|\}-\delta \\
= & -\max \left\{\left|H_{1}-H_{2}\right|,\left|H_{3}-H_{4}\right|\right\}-\delta
\end{aligned}
$$

and

$$
\begin{aligned}
A g(x)-A h(x)< & H(x, z, g(T(x, y)))-H(x, y, h(T(x, z)))+\delta \\
\leq & \max \{|H(x, y, g(T(x, y)))-H(x, y, h(T(x, y)))|, \\
& |H(x, z, g(T(x, z)))-H(x, z, h(T(x, z)))|\}+\delta \\
= & \max \left\{\left|H_{1}-H_{2}\right|,\left|H_{3}-H_{4}\right|\right\}+\delta,
\end{aligned}
$$

which yield that

$$
|A g(x)-A h(x)|<\max \left\{\left|H_{1}-H_{2}\right|,\left|H_{3}-H_{4}\right|\right\}+\delta .
$$

Similarly, we infer that (5.6) holds also for opt $\mathrm{t}_{y \in D}=\sup _{y \in D^{D}}$. Combining (5.2), (5.5) and (5.6), we arrive at

$$
\begin{aligned}
& \int_{0}^{|A g(x)-A h(x)|} \varphi(t) \mathrm{d} t \\
\leq & \int_{0}^{\max \left\{\left|H_{1}-H_{2}\right|,\left|H_{3}-H_{4}\right|\right\}+\delta} \varphi(t) \mathrm{d} t \\
= & \max \left\{\int_{0}^{\left|H_{1}-H_{2}\right|+\delta} \varphi(t) \mathrm{d} t, \int_{0}^{\left|H_{3}-H_{4}\right|+\delta} \varphi(t) \mathrm{d} t\right\} \\
= & \max \left\{\int_{0}^{\left|H_{1}-H_{2}\right|} \varphi(t) \mathrm{d} t+\int_{\left|H_{1}-H_{2}\right|}^{\left|H_{1}-H_{2}\right|+\delta} \varphi(t) \mathrm{d} t,\right. \\
\leq & \max \left\{\int_{0}^{\mid H_{1}-H_{2}} \varphi(t) \mathrm{d} t, \int_{0}^{\left|H_{3}-H_{4}\right|+\delta} \varphi(t) \mathrm{d} t\right\} \\
& +\max \left\{\int_{\left|H_{3}-H_{3}\right|}^{\left|H_{1}-H_{2}\right|+\delta} \varphi(t) \mathrm{d} t\right\} \\
\leq & \alpha\left(\| g-H_{4} \mid \int_{\mid}^{\left|H_{3}-H_{4}\right|+\delta} \varphi(t) \mathrm{d} t\right\}
\end{aligned}
$$

which means that

$$
\int_{0}^{\|A g-A h\|} \varphi(t) \mathrm{d} t \leq \alpha(\|g-h\|) \int_{0}^{\|g-h\|} \varphi(t) \mathrm{d} t+\varepsilon,
$$

letting $\varepsilon \rightarrow 0^{+}$in the above inequality, we deduce that

$$
\int_{0}^{\|A g-A h\|} \varphi(t) \mathrm{d} t \leq \alpha(\|g-h\|) \int_{0}^{\|g-h\|} \varphi(t) \mathrm{d} t .
$$

Thus, Theorem 5.1 follows from Theorem 3.1. This completes the proof. 
Remark 5.1. Theorem 5.1 extends and unifies Theorem 2.1 in [7], Theorem 3.1 in [18] and Theorem 3.2 in [25].

Theorem 5.2. Let $u: S \times D \rightarrow \mathbb{R}, T: S \times D \rightarrow S, H: S \times D \times \mathbb{R} \rightarrow \mathbb{R}, \phi \in \Phi$ and $\alpha$, $\beta: \mathbb{R}^{+} \rightarrow[0,1)$ satisfy (3.12), (5.1) and

$$
\begin{aligned}
& \int_{0}^{|H(x, y, g(T(x, y)))-H(x, y, h(T(x, y)))|} \varphi(t) \mathrm{d} t \\
& \leq \alpha(\|g-h\|) \int_{0}^{\|g-A g\|} \varphi(t) \mathrm{d} t+\beta(\|g-h\|) \int_{0}^{d(h, A h)} \varphi(t) \mathrm{d} t, \\
& \quad \forall(x, y, g, h) \in S \times D \times B(S) \times B(S) .
\end{aligned}
$$

Then the functional Equation (1.7) has a unique solution $w \in B(S)$ and $\left\{A^{n} z\right\}_{n \in \mathbb{N}}$ converges to $w$ for each $z \in B(S)$, where the mapping $A$ is defined by (5.3).

Proof. As in the proof of Theorem 5.1, by (3.12), (5.1), (5.3) and (5.7), we conclude that (5.4)-(5.6) hold and

$$
\begin{aligned}
& \int_{0}^{|A g(x)-A h(x)|} \varphi(t) \mathrm{d} t \\
& \leq \max \left\{\int_{0}^{\left|H_{1}-H_{2}\right|} \varphi(t) \mathrm{d} t, \int_{0}^{\left|H_{3}-H_{4}\right|} \varphi(t) \mathrm{d} t\right\} \\
& \quad+\max \left\{\int_{\left|H_{1}-H_{2}\right|}^{\left|H_{1}-H_{2}\right|+\delta} \varphi(t) \mathrm{d} t, \int_{\left|H_{3}-H_{4}\right|}^{\left|H_{3}-H_{4}\right|+\delta} \varphi(t) \mathrm{d} t\right\} \\
& \leq \alpha(\|g-h\|) \int_{0}^{\|g-A g\|} \varphi(t) \mathrm{d} t+\beta(\|g-h\|) \int_{0}^{\|h-A h\|} \varphi(t) \mathrm{d} t+\varepsilon,
\end{aligned}
$$

which yields that

$$
\begin{aligned}
\int_{0}^{\|A g-A h\|} \varphi(t) \mathrm{d} t \leq & \alpha(\|g-h\|) \int_{0}^{\|g-A g\|} \varphi(t) \mathrm{d} t \\
& +\beta(\|g-h\|) \int_{0}^{\|h-A h\|} \varphi(t) \mathrm{d} t+\varepsilon,
\end{aligned}
$$

letting $\varepsilon \rightarrow 0^{+}$in the above inequality, we infer that

$$
\begin{aligned}
\int_{0}^{\|A g-A h\|} \varphi(t) \mathrm{d} t \leq & \alpha(\|g-h\|) \int_{0}^{\|g-A g\|} \varphi(t) \mathrm{d} t \\
& +\beta(\|g-h\|) \int_{0}^{\|h-A h\|} \varphi(t) \mathrm{d} t .
\end{aligned}
$$

Thus, Theorem 5.2 follows from Theorem 3.2. This completes the proof.

Theorem 5.3. Let $u: S \times D \rightarrow \mathbb{R}, T: S \times \mathrm{D} \rightarrow S, H: S \times D \times \mathbb{R} \rightarrow \mathbb{R}, \phi \in \Phi$ and $\gamma: \mathbb{R}^{+} \rightarrow\left[0, \frac{1}{2}\right)$ satisfy (3.14), (5.1) and

$$
\begin{aligned}
& \int_{0}^{|H(x, y, g(T(x, y)))-H(x, y, h(T(x, y)))|} \varphi(t) \mathrm{d} t \\
& \leq \gamma(\|g-h\|)\left(\int_{0}^{\|g-A g\|} \varphi(t) \mathrm{d} t+\int_{0}^{\|h-A h\|} \varphi(t) \mathrm{d} t\right), \\
& \quad \forall(x, y, g, h) \in S \times D \times B(S) \times B(S) .
\end{aligned}
$$


Then the functional Equation (1.7) has a unique solution $w \in \mathrm{B}(S)$ and $\left\{A^{n} z\right\}_{n \in \mathbb{N}}$ converges to $w$ for each $z \in B(S)$, where the mapping $A$ is defined by (5.3).

Proof. As in the proof of Theorem 5.1, by (3.14), (5.1), (5.3) and (5.8), we conclude that (5.4)-(5.6) hold and

$$
\begin{aligned}
& \int_{0}^{|A g(x)-A h(x)|} \varphi(t) \mathrm{d} t \\
& \leq \max \left\{\int_{0}^{\left|H_{1}-H_{2}\right|} \varphi(t) \mathrm{d} t, \int_{0}^{\left|H_{3}-H_{4}\right|} \varphi(t) \mathrm{d} t\right\} \\
& \quad+\max \left\{\int_{\left|H_{1}-H_{2}\right|}^{\left|H_{1}-H_{2}\right|+\delta} \varphi(t) \mathrm{d} t, \int_{\left|H_{3}-H_{4}\right|}^{\left|H_{3}-H_{4}\right|+\delta} \varphi(t) \mathrm{d} t\right\} \\
& \leq \gamma(\|g-h\|)\left(\int_{0}^{\|g-A g\|} \varphi(t) \mathrm{d} t+\int_{0}^{\|h-A h\|} \varphi(t) \mathrm{d} t\right)+\varepsilon,
\end{aligned}
$$

which yields that

$$
\int_{0}^{\|A g-A h\|} \varphi(t) \mathrm{d} t \leq \gamma(\|g-h\|)\left(\int_{0}^{\|g-A g\|} \varphi(t) \mathrm{d} t+\int_{0}^{\|h-A h\|} \varphi(t) \mathrm{d} t\right)+\varepsilon,
$$

letting $\varepsilon \rightarrow 0^{+}$in the above inequality, we get that

$$
\int_{0}^{\|A g-A h\|} \varphi(t) \mathrm{d} t \leq \gamma(\|g-h\|)\left(\int_{0}^{\|g-A g\|} \varphi(t) \mathrm{d} t+\int_{0}^{\|h-A h\|} \varphi(t) \mathrm{d} t\right) .
$$

Thus, Theorem 5.3 follows from Theorem 3.3. This completes the proof.

\section{Acknowledgements}

The authors are indebted to the referees for their helpful comments.

\section{Author details}

${ }^{1}$ Department of Mathematics, Liaoning Normal University, Dalian, Liaoning 116029, People's Republic of China

${ }^{2}$ Department of Mathematics and RINS, Gyeongsang National University, Jinju 660-701, Korea

\section{Authors' contributions}

All authors contributed equally and significantly in writing this paper. All authors read and approved the final manuscript.

\section{Competing interests}

The authors declare that they have no competing interests.

Received: 13 April 2011 Accepted: 11 October 2011 Published: 11 October 2011

\section{References}

1. Banach, A: Sur les opérations dans les ensembles abstraits et leur application aux équations intégrales. Fund Math. 3, 133-181 (1922)

2. Aliouche, A: A common fixed point theorem for weakly compatible mappings in symmetric spaces satisfying a contractive condition of integral type. J Math Anal Appl. 322, 796-802 (2006). doi:10.1016/j.jmaa.2005.09.068

3. Altun, I, Türkoğlu, D: Some fixed point theorems for weakly compatible mappings satisfying an implicit relation. Taiwanese J Math. 13, 1291-1304 (2009)

4. Bellman, R: Dynamic Programming. Princeton University Press, Princeton (1957)

5. Bellman, R, Lee, ES: Functional equations arising in dynamic programming. Aequationes Math. 17, 1-18 (1978). doi:10.1007/BF01818535

6. Bhakta, PC, Choudhury, SR: Some existence theorems for functional equations arising in dynamic programming II. J Math Anal Appl. 131, $217-231$ (1988). doi:10.1016/0022-247X(88)90201-6

7. Bhakta, PC, Mitra, S: Some existence theorems for functional equations arising in dynamic programming. J Math Anal Appl. 98, 348-362 (1984). doi:10.1016/0022-247X(84)90254-3

8. Branciari, A: A fixed point theorem for mappings satisfying a general contractive condition of integral type. Int J Math Math Sci. 29, 531-536 (2002). doi:10.1155/S0161171202007524 
9. Djoudi, A, Aliouche, A: Common fixed point theorems of Greguš type for weakly compatible mappings satisfying contractive conditions of integral type. J Math Anal Appl. 329, 31-45 (2007). doi:10.1016/j.jmaa.2006.06.037

10. Jachymski, J: Remarks on contractive conditions of integral type. Nonlinear Anal. 71, 1073-1081 (2009). doi:10.1016/j. na.2008.11.046

11. Kamran, T: Mizoguchi-Takahashi's type fixed point theorem. Comput Math Appl. 57, 507-511 (2009). do::10.1016/j. camwa.2008.10.075

12. Kannan, R: Some results on fixed points. Bull Calcutta Math Soc. 60, 71-76 (1968)

13. Liu, Z: Coincidence theorems for expansion mappings with applications to the solutions of functional equations arising in dynamic programming. Acta Sci Math (Szeged). 65, 359-369 (1999)

14. Liu, Z: Compatible mappings and fixed points. Acta Sci Math (Szeged). 65, 371-383 (1999)

15. Liu, Z: Existence theorems of solutions for certain classes of functional equations arising in dynamic programming. Math Anal Appl. 262, 529-553 (2001). doi:10.1006/jmaa.2001.7551

16. Liu, Z, Agarwal, RP, Kang, SM: On solvability of functional equations and system of functional equations arising in dynamic programming. J Math Anal Appl. 297, 111-130 (2004). doi:10.1016/j.jmaa.2004.04.049

17. Liu, Z, Kang, SM: Properties of solutions for certain functional equations arising in dynamic programming. J Global Optim. 34, 273-292 (2006). doi:10.1007/s10898-005-2605-6

18. Liu, Z, Kang, SM: Existence and uniqueness of solutions for two classes of functional equations arising in dynamic programming. Acta Math Appl Sini (English Series). 23, 195-208 (2007). doi:10.1007/s10255-007-0363-6

19. Liu, Z, Kang, SM, Ume, JS: Solvability and convergence of iterative algorithms for certain functional equations arising in dynamic programming. Optimization. 59, 887-916 (2010). doi:10.1080/02331930902884182

20. Liu, Z, Ume, JS: On properties of solutions for a class of functional equations arising in dynamic programming. J Optim Theory Appl. 117, 533-551 (2003). doi:10.1023/A:1023945621360

21. Liu, Z, Ume, JS, Kang, SM: Some existence theorems for functional equations arising in dynamic programming. J Korean Math Soc. 34, 11-28 (2006)

22. Liu, Z, Ume, JS, Kang, SM: On properties of solutions for two functional equations arising in dynamic programming. Fixed Point Theory Appl 19 (2010). Article ID 905858

23. Liu, Z, Ume, JS, Kang, SM: Some existence theorems for functional equations and system of functional equations arising in dynamic programming. Taiwanese J Math. 14, 1517-1536 (2010)

24. Liu, Z, Wang, LL, Kim, HG, Kang, SM: Common fixed point theorems for contractive type mappings and their applications in dynamic programming. Bull Korean Math Soc. 45, 573-585 (2008). doi:10.4134/BKMS.2008.45.3.573

25. Liu, Z, Xu, YG, Ume, JS, Kang, SM: Solutions to two functional equations arising in dynamic programming. J Comput Appl Math. 192, 251-269 (2006). doi:10.1016/j.cam.2005.04.033

26. Liu, Z, Zhao, LS, Kang, SM, Ume, JS: On solvability of a functional equation. Optimization. 60, 365-375 (2011). doi:10.1080/02331930903121311

27. Mocanu, M, Popa, V: Some fixed point theorems for mappings satisfying implicit relations in symmetric spaces. Libertas Math. 28, 1-13 (2008)

28. Pathak, HK: Integral $\Phi$-type contractions and existence of continuous solutions for nonlinear integral inclusions. Nonlinear Anal. 71, e2577-e2591 (2009). doi:10.1016/j.na.2009.05.067

29. Rakotch, E: A note on contractive mappings. Proc Amer Math Soc. 13, 459-465 (1962). doi:10.1090/S0002-9939-19620148046-1

30. Suzuki, T: Meir-Keeler contractions of integral type are still Meir-Keeler contractions. Int J Math Math Sci 6 (2007). Article ID 39281

31. Hewitt, E, Stromberg, K: Real and Abstract Analysis. Springer, Berlin (1978)

doi:10.1186/1687-1812-2011-64

Cite this article as: Liu et al:: Fixed point theorems for mappings satisfying contractive conditions of integral type and applications. Fixed Point Theory and Applications 2011 2011:64.

\section{Submit your manuscript to a SpringerOpen ${ }^{\circ}$ journal and benefit from:}

Convenient online submission

- Rigorous peer review

- Immediate publication on acceptance

- Open access: articles freely available online

- High visibility within the field

- Retaining the copyright to your article

Submit your next manuscript at $\boldsymbol{s p r i n g e r o p e n . c o m ~}$ 